\title{
A new basal actinopterygian fish from the Anisian (Middle Triassic) of Luoping, Yunnan Province, Southwest China
}

Wen Wen, Qi-Yue Zhang, Shi-Xue Hu, Chang-Yong Zhou, Xie Tao, Jin-Yuan Huang, Zhong Qiang Chen, and Michael J. Benton

Acta Palaeontologica Polonica 57 (1), 2012: 149-160 doi: http://dx.doi.org/10.4202/app.2010.0089

The new neopterygian fish taxon Luoxiongichthys hyperdorsalis gen. et sp. nov. is established on the basis of five specimens from the second member of the Guanling Formation (Anisian, Middle Triassic) from Daaozi Quarry, Luoping, Yunnan Province, Southwest China. The new taxon is characterized by the following characters: triangular body outline with a distinct apex located between skull and dorsal fin; free maxilla; slender preopercular almost vertical; three suborbitals; at least eight strong branchiostegals with tubercles and comb-like ornamentation on the anterior margin; clavicles present; two postcleithra; ganoid scales covered by tubercles and pectinate ornamentation on the posterior margin with peg-and-socket structure; hemiheterocercal tail slightly forked. Comparison with basal actinopterygians reveals that the new taxon has parasemionotid-like triangular symplectics, but a semionotid opercular system. Cladistic analysis suggests that this new genus is a holostean, and either a basal halecomorph or basal semionotiform.

Key words: Actinopterygii, Halecomorphi,Triassic, Anisian, Luoping, Yunnan Province, China.

Wen Wen [wenwen2020240@163.com], Qi-Yue Zhang [yxzqy@sina.com],

Shi-Xue Hu [hushixue@hotmail.com], Chang-Yong Zhou [zhcy79@163.com], Xie

Tao [xt1982cd@163.com] , and Jin-Yuan Huang [huangjinyuancug@gmail.com

], Chengdu Institute of Geology and Mineral Resources, Chengdu 610081,

China; Zhong Qiang Chen [zqchen@cyllene.uwa.edu.au], Centre for

Petroleum Geoscience, University of Western Australia, 35 Stirling

Highway, Crawley, WA 6009, Australia; Michael J. Benton [mike.benton@ bristol.ac.uk], School of Earth

Sciences, University of Bristol, Bristol BS8 1RJ, UK (corresponding author).

This is an open-access article distributed under the terms of the Creative Commons

Attribution License (for details please see creativecommons.org), which permits unrestricted use, 
distribution, and reproduction in any medium, provided the original author and source are credited.

Faf Full text $(611.7 \mathrm{kB})$ 